\title{
Coding Criminal Justice Interactions with the MITI \\ Recommendations for Research and Practice
}

\author{
Scott T. Walters, $\mathrm{PhD}^{1}$, Malissa Cornett, $\mathrm{MPH}^{1}$, Amanda M. Vader, $\mathrm{MPH}^{1}$
}

\begin{abstract}
This article describes the coding portion of a study to test the effectiveness of a motivational interviewing (MI) training program for probation officers. We describe some of the challenges with using the Motivational Interview Treatment Integrity (MITI) instrument to code interactions between probation officers and clients. Our team of raters was able to obtain adequate inter-rater reliability on most MITI scales, though reliability ratings on some of the specific behavior counts such as Giving Information, MI Adherent, and MI Non-adherent fell considerably lower than the original MITI norming study. Our results suggest that the MITI is a mostly reliable instrument for coding criminal justice interactions, though there were exceptions to this rule. Based on our experiences, we discuss some of the ways that probation interactions might be different from traditional counseling interactions, and identify some rules of thumb that helped us to code interactions. We end with suggestions for how MITI feedback can be used effectively in training and supervision in criminal justice and other non-traditional settings.
\end{abstract}

Keywords

motivational interviewing, criminal justice, MITI, coding

A $\mathrm{s}$ motivational interviewing (MI) becomes more widely disseminated, there has been a need for standardized measures to assess MI performance The Motivational Interviewing Treatment Integrity (MITI 3.1; Moyers, Martin, Manuel, Miller, \& Ernst, 2009) coding system was designed to be used as a treatment integrity measure for clinical trials of $\mathrm{Ml}$, as well as a method of providing structured feedback to providers in non-research settings. The MITI evaluates global characteristics of the counseling session (i.e., Evocation, Collaboration, Autonomy-supportive [often grouped together as MI Spirit], Empathy, Direction) on a scale of 1-5, as well as specific counselor utterances (e.g., giving information, asking open and closed questions, offering simple and complex reflections, confronting [MI Nonadherent], affirming [MI Adherent]) that may be consistent or inconsistent with MI. The MITI manual also suggests threshold scores for evaluating MI competence; beginning proficiency is defined as at least 3.5/5.0 on the global ratings, a $1: 1$ ratio of reflections to questions, at least $50 \%$ open questions, and $40 \%$ complex reflections, whereas competency is defined as at least 4.0/5.0 on the global ratings, a 2:1 ratio of reflections

\footnotetext{
${ }^{1}$ At the time the research was conducted, the authors were affiliated with the University of Texas School of Public Health.

This project was funded through a cooperative agreement with the National Institute on Corrections (07C71GJS8, PI: Walters). The authors gratefully acknowledge the contributions of Steven Brazill, Ray Gingerich, Terri Thomas, and the 30 probation officers who helped to implement this project.
}

The authors have no conflict of interest to declare.

Correspondence concerning this article should be addressed to: Scott T. Walters, PhD, University of North Texas Health Science Center, 3500 Camp Bowie, EAD 711, Ft. Worth TX 76107. Email: Scott.Walters@UTSOUTHWESTERN.EDU to questions, at least $70 \%$ open questions, and $50 \%$ complex reflections.

Previous studies have found the MITI to be a reliable measure of MI skill, with inter-rater reliability ratings in the good-to-excellent range on most subscales (Bennett, Moore, et al., 2007; Bennett, Roberts, Vaughan, Gibbins, \& Rouse, 2007; Moyers, Martin, Manuel, Hendrickson, \& Miller, 2005; Pierson, et al., 2007; Thyrian, et al., 2007). One advantage of the MITI over simple clinical impression is that the MITI provides objective information on what are considered to be the most crucial aspects of MI. In addition, the MITI manual contains anchor scores and norms against which to compare clinician performance for purposes of supervision and quality control. Studies tend to find substantial agreement between patient and observer ratings of MI skill, and that certain aspects of MITI-rated performance predict better client outcome (Bennett, Roberts, Vaughan, Gibbins, \& Rouse, 2007; Pierson, et al., 2007; Tollison, et al., 2008). For instance, Tollison et al. (2008) found that the frequency of open questions and complex reflections both predicted drinking outcome in a group of heavy drinking college students. In other studies, global characteristics such as empathy positively predicted client change talk and a more favorable outcome (Boardman, Catley, Grobe, Little, \& Ahluwalia, 2006; Moyers, Miller, \& Hendrickson, 2005).

Originally, MI was conceived as a counseling interaction focused on a single target behavior, such as drinking, and it is in settings such as this that the MITI has mostly been normed. Despite a growing interest in using the MITI to code other kinds of interactions, such as healthcare, social work, or criminal justice interactions, there has been relatively little research on the reliability or validity of the MITI in these settings. In fact, we were unable to locate a single published study establishing the reliability of the MITI in criminal justice interactions. As we argue below, these settings may differ from more traditional counseling interactions in 
several respects, and pose difficulties for adequately coding and interpreting MITI scores.

This article draws from the coding portion of a randomized trial to test the effectiveness of $\mathrm{Ml}$ as a strategy for probation supervision in a large urban probation department. In the U.S., probation is the largest segment of the criminal justice system. Probation officers, the main contact for clients in the probation system, meet with clients to monitor progress, assess risk, and motivate clients to make changes that are consistent with conditions that have been specified by the Court. In this paper, we begin by briefly summarizing the results of our coding process. We then note some of the challenges we encountered when using the MITI to code probation officer interactions and offer recommendations for alterations in the MITI to improve its fit in criminal justice settings. We end with suggestions for how MITI feedback can be used effectively in training and supervision in criminal justice and other non-traditional settings.

\section{OVERVIEW OF THE PROJECT}

Enhancing Compliance and Officer Responsively (ENCORE) was a randomized effectiveness trial of $\mathrm{Ml}$ as a probation supervision strategy. The project has been described more extensively elsewhere (Walters, Vader, Nguyen, Harris, \& Eells, 2010). Briefly, 20 probation officers who were interested in receiving $\mathrm{Ml}$ training were randomized to MI-trained or Ml-untrained groups. The Ml training sequence for trained officers consisted of an initial two day training, followed by two half-day trainings and monthly supervision throughout a four month period during which officers submitted interview tapes for review and critique. The MIuntrained group did not receive any training during the study period. All officers completed two standardized role play interactions at three timepoints: baseline, two months, and six months. Each role play interaction (available from the authors upon request) described a medium-to-high risk probation case that involved compliance with substance abuse treatment, anger management, or other probation requirements. Officers were given a background and history on the case and instructed to conduct the interaction as if it were a real probation office visit.

Coding was conducted by three raters who were blind to study condition and timepoint. Training for the coders included an initial two day workshop consisting of practice tapes, videos, and manuscripts that focused on the coding protocol stated within the MITI manual. Both the senior author of this paper (STW) and lead coder (AMV) had previously completed a MITI training workshop with Dr. Theresa Moyers. Before coding actual project tapes, raters coded approximately ten practice tapes each over 60 days until adequate inter-rater reliability was obtained. Weekly meetings between the coders continued throughout the project, and a randomly selected $20 \%$ of tapes were coded by all coders. Intraclass correlation coefficients (ICCS) ranged from fair to excellent (Cicchetti, 1994; Shrout \& Fleiss, 1979). Table 1 shows how our ICC results compared to the original MITI reliability study conducted by Moyers et al. (2005). In general, while our global reliability ratings compared favorably to Moyers et al. (2005), some of the specific behavior counts such as Giving Information, MI Adherent, and MI Nonadherent fell considerably lower than the original study.

As described elsewhere (Walters et al., 2010), the Ml training sequence resulted in significant overall improvements in fidelity to $\mathrm{MI}$ compared to the group that did not receive the training. For instance, from baseline to 6 months, mean Empathy scores increased from 2.50 to 3.50 (out of 5.0) for the MI trained group compared to 2.31 to 1.79 for the untrained group; percent MI Adherent scores increased from 37.96 to $64.86 \%$ adherent for $\mathrm{Ml}$ trained group compared to 24.34 to $22.93 \%$ adherent for the untrained group. Other MITI indicators showed similar improvements that were mostly at, or just below recommended levels for beginning proficiency.

\section{CHALLENGES AND RECOMMENDATIONS FOR CODING MI IN PROBATION}

Our coders faced a number of challenges with respect to coding session tapes and interpreting scores. These difficulties most often resulted from the unique role that probation officers have, which balances helping and monitoring/enforcement tasks. Even though probation officers may be "change agents" in a broad sense, there are many individual tasks the officer must perform that are unrelated to motivating behavior change per se, including verifying probation progress, assessing risk, and delivering information and assistance. Because the MITI was originally designed to code single-behavior counseling interactions, our coders often had difficulty in three areas: 1) Coding and Interpreting Maintenance Tasks; 2) Accounting for Dual Roles; and 3) Identifying Target Behaviors. The sections below briefly describe the difficulties we encountered in each of these areas, and the approach we took to resolve the difficulties.

\section{Table 1}

Intraclass Correlation Coefficients in the Present Study and in Moyers, et al. (2005)

\begin{tabular}{|c|c|c|}
\hline & $\underline{\text { Present Study }}$ & Moyers et al. (2005) \\
\hline \multicolumn{3}{|l|}{ Global Ratings } \\
\hline Empathy & 0.492 & 0.518 \\
\hline MI Spirit & 0.677 & 0.585 \\
\hline \multicolumn{3}{|l|}{ Behavior Counts } \\
\hline Giving Information & 0.499 & 0.758 \\
\hline MI Adherent & 0.466 & 0.809 \\
\hline MI Non-adherent & 0.560 & 0.750 \\
\hline Closed Questions & 0.814 & 0.968 \\
\hline Open Questions & 0.832 & 0.939 \\
\hline Simple Reflections & 0.764 & 0.813 \\
\hline Complex Reflections & 0.654 & 0.576 \\
\hline
\end{tabular}




\section{Coding and Interpreting Maintenance Tasks}

One area of difficulty was the coding of maintenance tasks. Compared to other counseling interactions we have coded, our experience is that the probation system places many more clerical/documentation demands on probation officers. Some probation sessions are almost exclusively geared towards assessment tasks, while other sessions are partially geared toward assessment and partially geared toward behavior change. The difficulty we encountered was not the presence of such "housekeeping" tasks, but their sheer volume. In fact, the "directive" component of many sessions-the part the MITI was designed to code-often represented a minority of the session time. As one example, the probation system may require the office to ask a number of closed questions to verify current status and probation progress.

\section{$>$ Any change in your residence?}

$>$ Any contact with the victim?

$>$ Have you completed any community service hours since we last met?

Officers may also use a number of simple reflections to verify that they have heard the probationer correctly.

$>$ So, you're still living at the same place.

$>$ You have not had any contact whatsoever.

$>$ You've attended a few times. Have you gone at least twice a week?

In these cases, the purpose of the utterances is to verify rather than explore information, and thus closed questions and simple reflections may be adequate to the task. Closed questions were also sometimes used to obtain a simple yes/no response for documentation purposes. In some cases, a reflection (e.g., "You agreed to...") might be inadequate because it can be seen as putting words in a probationer's mouth, which can be insufficient for documentation. While these utterances are not difficult to code, and do not represent a violation of $\mathrm{Ml}$ per se, the frequency of such utterances can make summary scores difficult to interpret. Likewise, in the case of simple reflections it can appear that the officer is not making much of an attempt to explore the client's perspective, when in fact, simple reflections have been used appropriately to make sure the officer understands the factual information the probationer has provided. The danger is that summary MITI scores may not adequately distinguish between officers who are using appropriate skills on Ml-irrelevant tasks, and those who are performing badly on tasks where MI may be appropriate. In fact, the MITI was designed to rate counseling interactions focused on change in a target behavior, and thus some of the suggested competency thresholds for questions and reflections may not be reasonable for interactions that are more focused on assessment or verification tasks. While we were reluctant to disregard the thresholds entirely, we think that it is important to keep in mind the specific goals of the interview when providing feedback to officers. In fact, some probation interactions look very much like counseling interactions (where we might expect the MITI thresholds to apply), while others look much more like assessment interviews (where the MITI might be inappropriate).

One solution to this problem in our supervision sessions with the MI trained officers was to ask them to submit only tapes of meetings that were definitely focused on behavior change. (For the coding portion of the project described in this paper, we created cases that were specifically focused on probationers who were ambivalent about some area of behavior change.) This eliminated many interactions that were focused primarily on assessing or verifying progress, and many other (usually brief) interactions in which probationers were making good progress. Another strategy in our supervision sessions was to ask officers to conduct "housekeeping" tasks early in the session, so that MI coding could begin when the task shifted from verification of current information to talking about behavior change. This made it easier for coders to see the sections where MI would be relevant to the interaction. However, in practice, both of these strategies were difficult to achieve. Some officers preferred to move topically, verifying progress and then talking about change in one area, before moving to the next. So directive tasks (where Ml may be appropriate) become more intertwined with housekeeping tasks (where MI may be irrelevant).

Another option for future coding studies like this might be to make changes to the MITI itself to accommodate interactions that contain more substantial maintenance components. As an example, one widely used system for coding doctor-patient interactions, the Roter Interaction Analysis System (RIAS; http://www.rias.org) contains many more categories for coding information-gathering tasks. (The RIAS captures 34 categories of physician communication, including tonal qualities and global affect.) While such coding systems lack the motivational features of the MITI, they would better capture some of the assessment and verification tasks that are expected of social workers, probation officers, and healthcare workers. The RIAS is organized around the four major sections of most medical visits-opening, history taking, exam, counseling, and closing, and it may be that $\mathrm{Ml}$ strategies are more relevant during some sections than others.

In terms of the specific task, a hybrid version of the MITI might distinguish between closed questions that are intended to verify current information (e.g., "You've moved since we last spoke, right?"), check for understanding (e.g., "Did I get that right?"), or explore future behavior (e.g., "Are you going to start on your community service this week?"). Likewise, an information-giving category might distinguish between information that is intended to inform about what the officer will do (e.g., "I'll file this petition with the court on your behalf.") vs. what the probationer is expected to do ("When you get your copy in the mail, you will need to sign and return it."). Indeed, in the current system it is sometimes difficult to distinguish between utterances that are MI Nonadherent vs. those that are Ml-irrelevant. This depends on the specific language and tone used, but also on the context of the utterance. Allowing for a range of categories within the MITI might provide a more accurate picture of the flow of the session, and help supervisors and researchers to understand how utterances contribute to the overall flow of the session.

\section{Accounting for Dual Roles}

Another area of difficulty was in determining how directions and information-giving contributed to the overall global ratings of the session. As discussed above, the global rating on Evocation can be considerably compromised when a substantial portion of the session is focused on asking questions to verify progress or giving (rather than eliciting) information. This interpretation is complicated by the inherent power difference and rigidity of some parts of the criminal justice interaction. In some ways, probation officers may have the flexibility to talk with a person about how/when they will complete requirements or emphasize the person's choice in completing requirements; but in other ways, a probation officer may have to be very rigid in terms of the specific requirements that have been dictated by the court.

As one example, in a traditional counseling interaction, the counselor may have much more flexibility to talk in terms of the client's stated interests or goals. For instance, moderate drinking may be a legitimate (however unwise) goal for clients who have chosen that outcome. But in a criminal justice context, moderate drinking is a more complicated topic if the court has mandated that the client remain abstinent. Although the officer can strongly support the client's right to choose to drink moderately, he/she must report any instances of drinking 
to the supervising court. Likewise, the set of court mandates (and the officer's responsibility to monitor the probationer's progress) may also narrow the range of solutions that will be acceptable, for instance if a probationer wants to participate in outpatient treatment when the court has dictated inpatient treatment.

Because of the presence of court mandates, it was sometimes difficult for coders to determine how specific utterances contributed to the overall autonomy or evocation of the session. For instance, when a probation officer says that a client "needs to" attend AA meetings or "should" refrain from drinking it was sometimes unclear whether these utterances should be seen as providing information about court mandates or as MI Non-adherent confrontational statements. In fact, the areas in which our ICCs fell short in comparison to Moyers et al. (2005) tended to be concentrated around the issue of information giving and whether an utterance was MI Adherent or Non-adherent. In some instances, the tone of the utterance or very small differences in phraseology provided the only clues as to how it should be coded. Consider the following probation officer utterances, where the probationer has been court mandated to attend AA:

> You have a problem and need to attend AA. (MI Non-adherent; confront, direct)

$>$ As part of your probation, you'll need to attend AA. (Probably MI Non-adherent; direct)

> If you want to avoid problems with your probation, you'll need to attend $A A$. (Probably not MI Non-adherent; action contingent on desire)

> Your court conditions state that you need to attend AA. What do you want to do about that? (not MI Non-adherent; does not dictate an action)

The first two statements would probably be coded as MI Nonadherent (and reflect negatively on autonomy) because they seem to suggest that the probationer does not have a choice-the probationer must attend AA. The second two statements would probably be coded as giving information (and be seen as autonomy-neutral), because they suggest that an action is conditional on the desire of the client-if the probationer wants to be successful. There were many statements like the second and third that fell into the "gray" range; although we developed rules around coding such utterances, it was still very difficult to reach agreement when coding independently.

Another difficult situation was when probationers reported behavior that violated their probation conditions. In such situations, it is generally not an option for the officer to leave such reports unaddressed. The officer must report illicit behavior to the court and must inform the probationer that he/she will do so.

Probationer: I just used a little bit of weed to take the edge off. But l'm done with that.

Officer: We talked earlier on about my dual role.... both to you and the court. Because of that, I will need to report that to the court, and if there are any further instances of drug use, you will likely be looking at a jail sanction.

Outside of a criminal justice context, such an exchange might be coded as an MI Non-adherent confrontational statement, because it seems to dictate a course of action for the probationer; however in this context, the officer has been true to his/her duties as an agent of the court. In this instance, we would be more likely to code such an exchange as giving information because the officer has informed the probationer about what the officer must do and what might happen if future drug use is discovered, while at the same time not prescribing a course of action for the probationer.
Finally, we encountered many miscellaneous instances of information-giving during more extensive assessment interviews.

You'll need to have a valid form of identification to get into the program. If you don't have a license, you can get one from the Department of Motor Vehicles.

According to the MITI manual, such unsolicited information might be seen as lack of power sharing and thus might decrease the global rating for Collaboration. However, in this instance, the officer might have been trying to help the probationer understand and interpret the court requirements, which involved, in this case, obtaining an identification card. Again, the difficulty was not their presence, but the overall volume of such informational/teaching statements, compared to longer counseling sessions. For our coding project, we asked coders to consider the transaction as a whole and decide whether the officer was restating and interpreting the court conditions to make sure the probationer was aware of them (autonomy neutral) or using court conditions to lessen the probationer's perception of control (autonomy diminishing).

\section{Determining a Target Behavior}

Because probation officers must often focus on several target behaviors, in some instances it was difficult to determine global ratings for Collaboration and Direction. Successful collaboration requires mutual problem solving and planning between the officer and the probationer to remedy a target behavior. However, when there are multiple behaviors being addressed, as there are frequently in a probation setting, problem solving and planning can prove to be more difficult for the officer as he/she is having to change direction, or focus, on the behavior at hand.

\section{Officer: So you were drinking when you hit your wife? \\ Offender: I drink every day, and sometimes she says things that make me angry.}

In this example, the officer has identified three potential target behaviors-substance abuse, spousal abuse, and anger management--each of which may warrant a different conversation, and only some of which may be related to probation requirements per se. It is not always clear which behavior warrants more attention as they all need to be addressed at some point in the interview. Thus, it is difficult to isolate the true target behavior in the session.

In our study, we asked coders to consider the gestalt of the session, and interpret the target behavior broadly as making positive steps on behaviors that would increase success on probation conditions. In some instances, this included multiple behaviors that might be related to probation success.

\section{RECOMMENDATIONS FOR USE OF THE MITI FOR TRAINING AND PRACTICE IN PROBATION}

Although we did encounter some difficulties in coding the language of probation sessions, the results of the parent study show that the MITI was a reliable instrument and that the MI-trained group did increase their mean MITI ratings when compared to the untrained group. This suggests that probation officers can be taught to use MI skills in their interactions with probationers, at least during relatively controlled role-play conditions. Nonetheless, in addition to the more global tone of the session, there were a number of specific behaviors that frequently occurred that caused officers to be rated more poorly even when it appeared that the officer was trying to engage or support the client. 
In some cases, there was a clear violation of the MI style indicators, as evidenced by harsh, confrontational statements (e.g., "What did you expect the cops to do? You were found with the knife!") or copious advice giving (e.g., "Why don't you try talking to your wife about it? You've got to share this with her."). However, the more common difficulties had to do with instructions about future behavior or clarifications of probation conditions that were coded as confrontational statements or unsolicited advice because of the way the utterance was stated.

For instance, our coders agreed that the following statements were likely to be coded as MI Non-adherent even when the probation officer assumed a neutral tone of voice and generally positive spirit. All were judged to be directive per the MITI manual.

1. You did very well last semester. I want you to apply yourself to school that same way.

2. I need you to fill out your monthly report form today.

3. Call and let me know how your job interview goes.

4. Don't forget to call.

5. Behave this weekend, but have fun.

Officers who were able to communicate such information in an Ml adherent way most often used strategies such as removing the first person pronouns from their utterances, asking questions rather than telling what to do, and deferring to court requirements or policy. For instance, our coders agreed that the following statements conveyed very similar information, while generally steering clear of MI Non-adherent (directive) language.

1. You did very well last semester. What are some of the things you could do to keep your grades up?

2. Would you please fill out your monthly report today?

3. I would love to hear how your job interview goes. Would you mind calling to let me know how it goes?

4. Just a reminder that the deadline for registration is approaching, so if you want to get enrolled in the class, you'll need to call this week.

5. I hope you have a fun, safe time this weekend. (Or, What are some things you could do to stay safe this weekend?)

\section{CONCLUSION}

This paper described some of the challenges of using the MITI to code criminal justice interactions and some of the ways we resolved these difficulties. While we did not make changes to the MITI instrument itself, the rules of thumb we describe above may help to identify the strengths and weaknesses of the MITI in coding criminal justice interactions. Importantly, we found the MITI was already a useful and reliable coding instrument in most areas. Rather, the challenges of MITI coding most often resulted from the unique tasks of probation officers.

As a result of our coding experiences, we have made a series of changes to our officer trainings. Part of our training now focuses on the overall $\mathrm{Ml}$ spirit indicators, as evidenced by probation officers' attentive, respectful attitude. Open questions and reflections are often indicators of such an interest and respect. Another part of the training involves an attention to phraseology to help officers bring some statements more in line with the technical aspects of MI that are likely to be captured on the MITI utterance ratios. Reviewing session tapes often involves an explanation of why a statement-though well intentioned-would be coded as MI Non-adherent, as well as brainstorming alternative ways of communicating such information. We hope that this balance of style and content will help officers not only to exhibit the spirit of MI, but also to be able to perform well on Ml rating instruments. Most importantly, we are hopeful that these small changes in our training curriculum will improve the communication between officers and clients, and contribute to a more positive and helpful experience for clients on probation.

\section{REFERENCES}

Bennett, G. A., Moore, J., Vaughan, T., Rouse, L., GibbinS, J. A., Thomas, P., et al (2007). Strengthening Motivational Interviewing skills following initial training: A randomised trial of workplace-based reflective practice. Addictive Behaviors, 32, 2963-2975.

Bennett, G. A., Roberts, H. A., Vaughan, T. E., Gibbins, J. A., \& Rouse, L. (2007) Evaluating a method of assessing competence in Motivational Interviewing: A study using simulated patients in the United Kingdom. Addictive Behaviors, $32,69-79$.

Bennett, G. A., Roberts, H. A., Vaughan, T. E., Gibbins, J. A., \& Rouse, L. (2007). Evaluating a method of assessing competence in Motivational Interviewing: A study using simulated patients in the United Kingdom. Addictive Behaviors, 32, 69-79.

Boardman, T., Catley, D., Grobe, J. E., Little, T. D., \& Ahluwalia, J. S. (2006). Using motivational interviewing with smokers: Do therapist behaviors relate to engagement and therapeutic alliance? Journal of Substance Abuse Treatment, 31, 329-339.

Cicchetti, D. V. (1994). Guidelines, criteria, and rules of thumb for evaluating normal and standardized assessment instruments in psychology. Psychological Assessment, 6, 284-290.

Madson, M. B., \& Campbell, T. C. (2006). Measures of fidelity in motivational enhancement: A systematic review. Journal of Substance Abuse Treatment, 31, 67-73.

Moyers, T. B., Martin, T., Manuel, J. K., Hendrickson, S. M., \& Miller, W. R. (2005). Assessing competence in the use of motivational interviewing. Journal of Substance Abuse Treatment, 28, 19-26.

Moyers, T. B., Martin, T., Manuel, J. K., Miller, W. R., \& Ernst, D. (2009). Revised global scales: Motivational interviewing treatment integrity 3.1 (MITI 3.1). Retrieved from http://casaa.unm.edu/download/miti3_1.pdf

Moyers, T. B., Miller, W. R., \& Hendrickson, S. M. L. (2005). How does Motivational Interviewing work? Therapist interpersonal skill as a predictor of client behavior within Motivational Interviewing sessions. Journal of Consulting and Clinical Psychology, 73, 590-598.

Pierson, H. M., Hayes, S. C., Gifford, E. V., Roget, N., Padilla, M., Bissett, R., et al. (2007). An examination of the Motivational Interviewing Treatment Integrity code. Journal of Substance Abuse Treatment, 32, 11-17.

Shrout, P. E., \& Fleiss, J. L. (1979). Intraclass correlations: Uses in assessing rater reliability. Psychological Bulletin, 86, 420-428.

Thyrian, J. R., Freyer-Adam, J., Hannover, W., Roske, K., Mentzel, F., Kufeld, C., et al. (2007). Adherence to the principles of Motivational Interviewing, clients' characteristics and behavior outcome in a smoking cessation and relapse prevention trial in women postpartum. Addictive Behaviors, 32, 2297-2303.

Tollison, S. J., Lee, C. M., Neighbors, C., Neil, T. A., Olson, N. D., \& Larimer, M. E. (2008). Questions and reflections: The use of motivational interviewing microskills in a peer-led brief alcohol intervention for college students. Behavior Therapy, 39, 183-194. 
Walters, S. T., Vader, A. M., Nguyen, N. D., Harris, T. R., \& Eells, J. (2010).

Motivational interviewing as a supervision strategy in probation: A andomized

effectiveness trial. Journal of Offender Rehabilitation, 24(11), 309-323.

This work is licensed under a Creative Commons AttributionNoncommercial-No Derivative Works 3.0 United States License.

This journal is published by the University Library System of the University of Pittsburgh as part of its D-Scribe Digital Publishing Program, and is cosponsored by the Motivational Interviewing Network of Trainers. 